\title{
Textiloma intraluminal: Complicación tardía de una cirugía intraabdominal
}

\section{Intraluminal Textiloma: Late Complication of Intra-abdominal Surgery}

Gaspar A. Motta-Ramírez ${ }^{1 *}$, Jorge E. Aguilar-Garibay², Oliva González-Burgos ${ }^{3}$,

Cristian Espinoza-Ramírez ${ }^{4}$ y Pablo F. Pérez-Barbosa ${ }^{4}$

${ }^{1}$ Jefatura Regional Sanidad Militar, XII R.M, Irapuato, Guanajuato; ${ }^{2}$ Servicio de Cirugía, Hospital Militar de Zona, Cuernavaca, Morelos; ${ }^{3}$ Departamento de Radiología e Imagen, Hospital Militar Regional y de Especialidades, Guadalajara, Jalisco; ${ }^{4}$ Servicio de Cirugía, Hospital Militar Regional y de Especialidades, Guadalajara, Jalisco. México

\section{RESUMEN}

La compresa quirúrgica retenida es el resultado de un error quirúrgico poco frecuente y evitable, que representa un serio problema que origina una amplia variedad de cuadros clínicos y puede causar graves complicaciones, que pueden incluso ocasionar la muerte. Se trata de un paciente masculino de 41 años, quien acudió por un síndrome doloroso abdominal, de subagudo a crónico, con antecedente quirúrgico 3 años antes. Durante la laparotomía, se definió a nivel yeyunal una masa intraluminal de aproximadamente $30 \mathrm{~cm}$ de longitud que se manejó con enterotomía y se extrajo una compresa.

Palabras clave: Textiloma. Gossypiboma. Compresa quirúrgica retenida.

\section{ABSTRACT}

The retained surgical compress or textiloma -removed surgical material after abdominal surgery- is the result of a rare and avoidable surgical error, represents a serious problem and causes a wide variety of clinical symptoms included serious complications that can lead to death. We present a male 41-year-old patient who presented for abdominal pain syndrome, from subacute to chronic, with a surgical history 3 years previously. During the laparotomy, an intraluminal mass of approximately $30 \mathrm{~cm}$ in length was defined at the jejunal level, which is managed with enterotomy by extracting a compress.

Keys words: Textiloma. Gossypiboma. Retained Surgical Sponge. 


\section{PRESENTACIÓN DEL CASO CLÍNICO}

Paciente masculino de 41 años, originario de Oaxaca, que vive en Zapopan, Jalisco, sin enfermedades crónico-degenerativas, con antecedente de laparotomía exploratoria secundaria a un trauma por herida por proyectil de arma de fuego en 2015, que condicionó una resección intestinal y anastomosis. Se desconoce el segmento resecado y el tipo de anastomosis, así mismo, uno de los proyectiles condicionó la fractura del ala del hueso iliaco izquierdo.

Acudió al servicio de urgencias, y refirió 2 días de evolución con dolor (intensidad 7/10) en el hipocondrio derecho después de la ingesta de colecistoquinéticos, de tipo cólico, sin irradiaciones, acompañado de náuseas y en dos ocasiones de vómito de contenido biliar, y de distensión abdominal. Ese día, a su ingreso, tuvo una evacuación de características normales.

A la exploración física presenta taquicardia (103 lpm). El resto de signos están dentro de los parámetros normales; consciente, orientado, mucosas hidratadas, tegumentos pálidos, exploración cardiopulmonar sin alteraciones: abdomen semigloboso a expensas de la distensión, con cicatriz en la línea media por antecedente quirúrgico, ruidos intestinales presentes, de buena intensidad y frecuencia, sin masas palpables, con dolor a la palpación en el hipocondrio y el marco cólico derechos, Murphy positivo, maniobras apendiculares negativas, sin datos de irritación peritoneal.

Se realizan estudios de laboratorio en urgencias tanto biometría hemática y examen general de orina mismos que se reportan dentro de los limites de la normalidad.

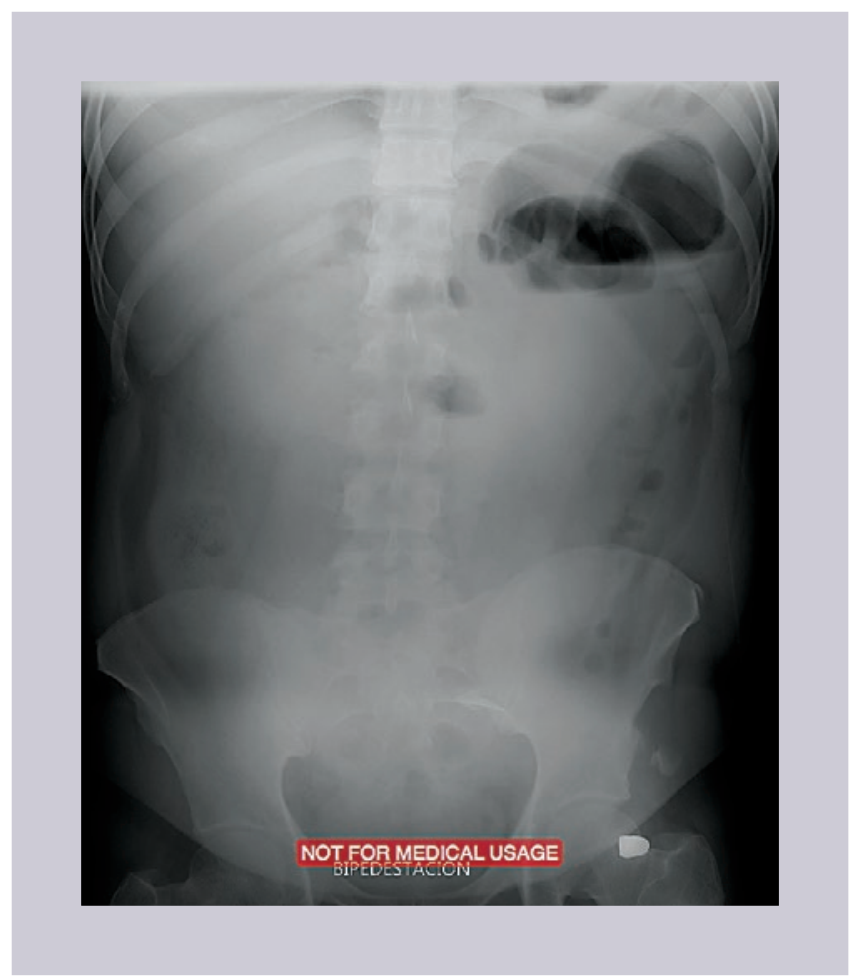

Figura 1. Proyección abdominal en la que se observa un asa distendida en el hemiabdomen izquierdo, con gran cantidad de material fecal en el colon derecho y aire distal sin niveles hidroaéreos. No se define material radiopaco intrabdominal.

Dentro del abordaje se realizó una radiografía de tórax, que se encontró dentro de los parámetros normales y en la proyección abdominal (Fig. 1) se observó un asa distendida en el hemiabdomen izquierdo, con gran cantidad de materia fecal en el colon derecho y aire distal sin niveles hidroaéreos. También se le realizó un ultrasonido del hígado y de las vías biliares, que informó de litiasis vesicular, bilis litogénica del 70\% e hidrocolecisto.

Se estableció el diagnóstico de cólico vesicular secundario a litiasis vesicular, con anemia microcítica e hipocrómica, por lo que se decidió la hospitalización del paciente para el control del dolor y el estudio del cuadro anémico. Durante su primer día de estancia hospitalaria se solicitó sangre oculta en heces, que resultó 
positiva. Clínicamente, las constantes vitales estaban dentro de los parámetros normales. El paciente refirió cese del dolor, a lo que se agregó evacuaciones semilíquidas y mejoría de la distensión. A la exploración abdominal presenta ruidos intestinales aumentados, dolor a la palpación en el hipocondrio derecho, sin masas palpables y sin datos de irritación peritoneal.

Se inició dieta líquida por la mañana, que toleró adecuadamente, y se progresó a dieta blanda, con vómito en una ocasión, por lo que se suspendió la vía oral. Tuvo un pico febril de $38.3^{\circ} \mathrm{C}$ por la noche, lo que motivó que se iniciara tratamiento con antibiótico.

Al segundo día del ingreso, el paciente refirió aumento del dolor abdominal. Clínicamente, presentaba taquicardia (100 lpm), con las constantes vitales dentro de los parámetros normales, distensión abdominal importante, ruidos intestinales presentes, aumentados, sin masas palpables, con dolor a la palpación en el marco cólico derecho y dolor leve a la descompresión, por lo que se decidió colocar una sonda nasogástrica, en la que se obtuvo un gasto fecaloide de 1.000 cc, aproximadamente.

Se abordó el escalón inmediato superior y se le realizó un estudio de imagen con una tomografía computarizada (TC) abdominopélvica, bajo la sospecha de síndrome de obstrucción intestinal (SOI), y en la que se identificó obstrucción intestinal mecánica baja, yeyunal, secundaria a un cuerpo extraño intraluminal de aspecto textil (Fig. 2).

Se decidió realizar laparotomía exploratoria y se encontraron múltiples adherencias laxas, y a nivel de yeyuno se palpó una masa intraluminal de aproximadamente $30 \mathrm{~cm}$ de longitud que se manejó con enterotomía a ese nivel y se extrajo una compresa (Fig. 3). Por el estado inflamatorio intestinal se decide realizar una resección del segmento intestinal y anastomosis término terminal.

El paciente ingresa a la Unidad de Terapia Intensiva de Adultos (UTIA) para su recuperación posoperatoria, donde cursa sus primeros días con datos de respuesta inflamatoria, que condicionó la falla orgánica múltiple. Se realizó un manejo agresivo, y a las 48 horas presentó mejoría. Posteriormente evolucionó con neumonía asociada a ventilador, por lo que se inició una pauta con tratamiento antibiótico doble. Al día 5 de la pauta, cursó hacia la mejoría y salió de la UTIA a los 15 días.

En sala evolucionó hacia la mejoría, y una semana después fue dado de alta para control como externo.

\section{DISCUSIÓN}

La retención de compresas intraperitoneales, gossypiboma, textiloma, etc. puede no ser considerada como la sospecha clínica inicial que explique el cuadro clínico del paciente, pero dicha situación puede ser reconocida por los estudios de imagen ${ }^{1}$.

El diagnóstico de textiloma representa un reto para los médicos, debido a lo inespecífico de sus manifestaciones clínicas, la multiplicidad de apariencias radiológicas y de imagen y a lo infrecuente que es el considerar esta posibilidad diagnóstica ${ }^{2}$.

La retención de compresas ocurre más comúnmente en la cavidad abdominal o pélvica, generalmente después de cirugías abdominales 

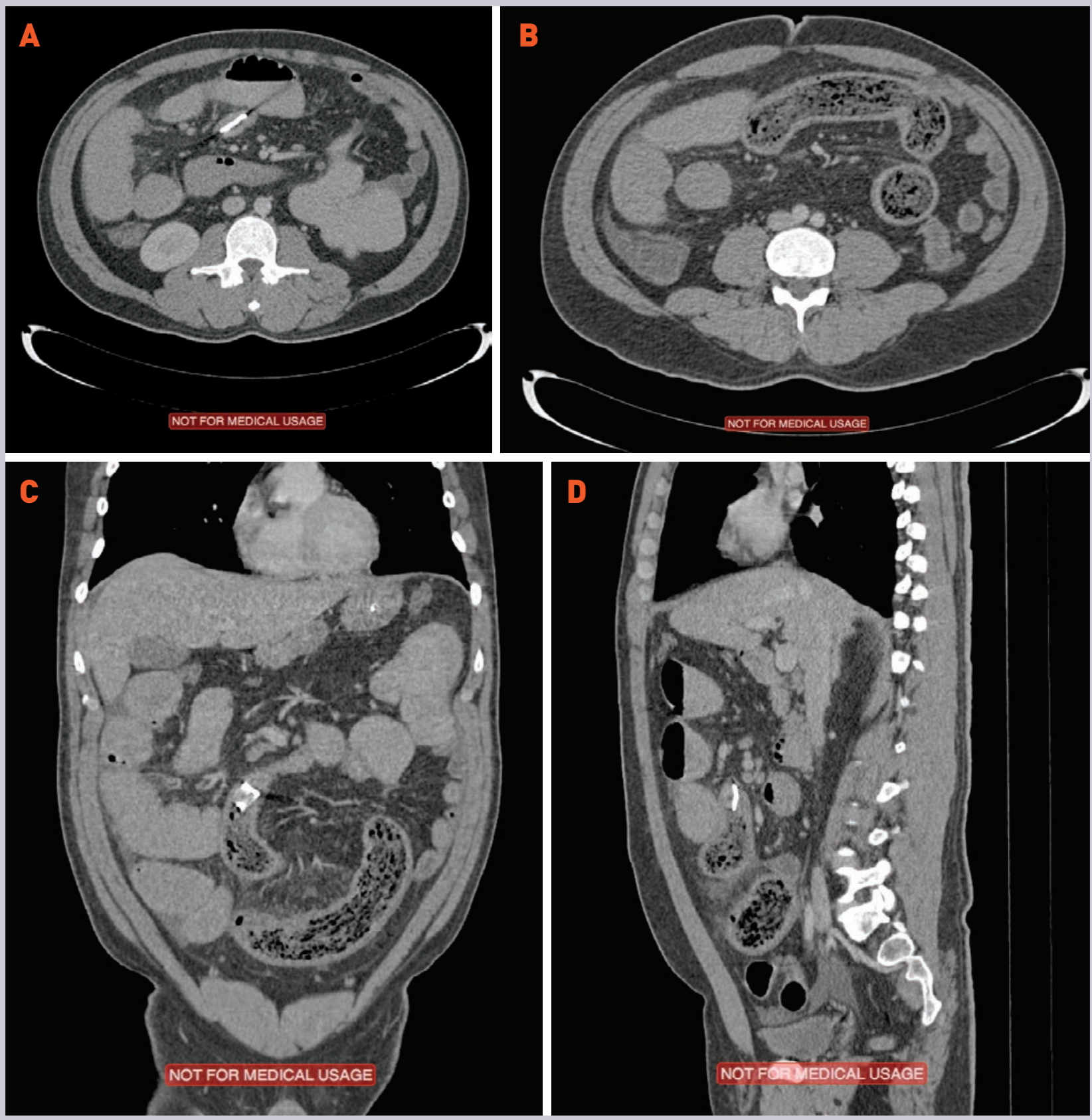

Figura 2. Selección de imágenes de una tomografía computarizada abdominopélvica simple bajo la indicación de sospecha de síndrome de obstrucción intestinal, y en la que se identifico: A: imagen axial con material denso, de densidad metálica, intraluminal. B: imagen axial, con el signo de fecalización del contenido intraluminal y obstrucción intestinal mecánica baja, yeyunal, secundaria a un cuerpo extraño intraluminal de aspecto textil. C: Reconstrucción multiplanar (RMP) coronal. D: Reconstrucción multiplanar (RMP) sagital. C y D definen el sitio de obstrucción secundario al cuerpo extraño intraluminal. 


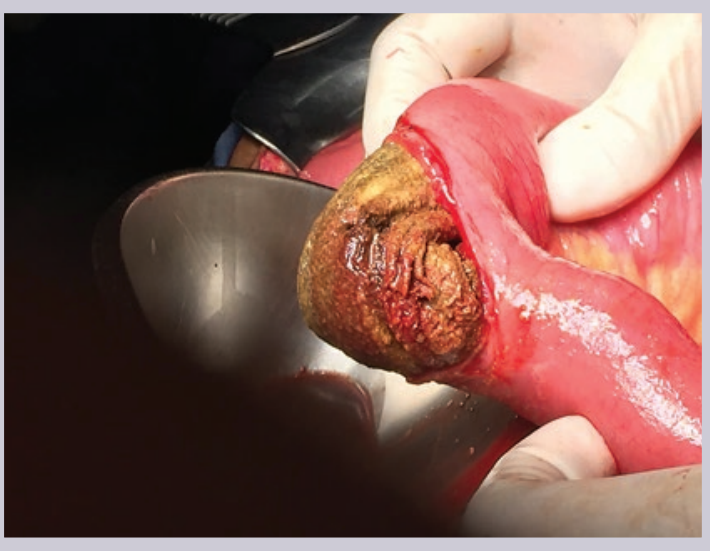

Figura 3. Foto quirúrgica de enterotomía yeyunal en el sitio donde se definió a la palpación una masa intraluminal de aproximadamente $30 \mathrm{~cm}$ de longitud, y de donde finalmente se extrajo una compresa.

superiores o ginecológicas. La mayoría de los textilomas son identificados solo después de la cirugía, y las técnicas para el diagnóstico por imagen, en ocasiones, son de gran ayuda, como en el caso que nos ocupa. Se ha señalado que tales técnicas para el diagnóstico por imagen son inespecíficas ${ }^{1}$. En nuestra opinión, lo que sucede es que el índice de sospecha de la posibilidad diagnóstica es muy bajo y por ello el cuerpo extraño puede pasar inadvertido. También se produce el hecho desafortunado de que un número importante de estudios no son evaluados por expertos, médicos especialistas en radiología e imagen. Un alto índice de sospecha asociado a los hallazgos de imagen es esencial para establecer a tiempo el diagnóstico ${ }^{2}$.

La identificación y demostración del marcador radiopaco representa una señal importante que debe de identificarse y que, gracias al uso de la TC, fue fácilmente identificable. El marcador radiopaco llega a ser evidente cuando el estudio radiológico, proyección abdominal, es utilizado para el abordaje clínico-diagnóstico inicial. El marcador radiopaco es, sin duda, un hallazgo sine quanon que deberá intencionadamente ser buscado ${ }^{2}$.

En referencia a la incidencia de este tipo de complicaciones, es una tarea laboriosa debido a las implicaciones médico-legales inherentes, ya que la mayoría de casos no se publican, por lo que la magnitud del problema es difícil de apreciar. De ahí, el interés en la publicación de este tipo de patología, en un afán de generar la cultura y la aceptación del error médico ${ }^{2,3}$.

La compresa quirúrgica retenida es causa de complicaciones graves, como perforación intestinal, obstrucción, fístulas, sepsis o muerte. Puede migrar dentro del estómago, íleon, recto, colon o vejiga, sin apertura aparente de la pared de estos órganos luminales. Se han descrito ubicaciones poco habituales, como los músculos paraespinales, la región torácica, los miembros inferiores y el espacio pericárdico. Son más comunes en pacientes obesos, ya que tienen amplios espacios intraperitoneales donde las compresas se pueden olvidar y pasar desapercibidas. La obesidad puede incrementar las dificultades técnicas ${ }^{1}$.

El cuadro clínico puede ser variable, y su presentación aguda aparecerá como una complicación inmediata de una cirugía, con predominio de la respuesta exudativa, con frecuente formación de abscesos y fístulas cutáneas. Sin embargo, predomina la presentación clínica a largo plazo, que puede condicionar síntomas durante meses, o incluso años, después de la intervención quirúrgica, y las manifestaciones clínicas son sugerentes de un proceso ocupativo expansivo tumoral abdominal ${ }^{2}$ y/o de cuadros de obstrucción/pseudoobstrucción intestinal. 
Respecto al textiloma en la cavidad abdominal, la evolución más frecuente es hacia la formación de fístulas digestivas, debido a un mecanismo de defensa, en que el propio organismo intenta eliminar el cuerpo extraño. Estas migraciones pueden producir diferentes problemas relacionados con un proceso séptico local que provoca la fístula y posterior migración del textiloma.

La TC es la mejor técnica de imagen para evaluar las cavidades abdominal y pélvica en pacientes con patología abdominal conocida en los que se sospecha trastornos inflamatorios, procesos ocupativos de origen benigno y/o maligno, así como en trauma abdominal. La TC permite evaluar patrones normales, variantes $\mathrm{y}$ condiciones anormales, incluidos aquellos de atenuación del tracto gastrointestinal. Asimismo, evalúa el engrosamiento de la pared y sus características (si es focal, segmentario o difuso o con compromiso del mesenterio). Hoy en día, todas las anormalidades son más evidentes gracias a los equipos multicorte, el equipo dual, las estaciones de posproceso con reconstrucciones 2D (multiplanares), máxima intensidad de proyección y 3D, la angiotomografía, etc. ${ }^{4}$

La pared del intestino delgado es delgada (entre 1 y $2 \mathrm{~mm}$ ) cuando la luz está bien distendida, y puede no definirse durante el estudio de la TC. Algunos autores dan como límite superior normal la medición de la pared de hasta 2 a 3 $\mathrm{mm}^{4}$. Una medición por arriba de esos valores es indicativa de proceso inflamatorio que afecta no solo el grosor de la pared sino también, por extensión, la grasa perilesional. La TC define el contenido intraluminal y facilita la identificación de cuerpos extraños, como agente causal del SOI, como es el caso que nos ocupa.

El SOI, acompañado de dolor abdominal agudo, representa una causa frecuente de hospitalización y de consulta quirúrgica. El SOI se sospecha en la exploración física; sin embargo, tanto clínicamente como por laboratorio su presentación es inespecífica. Por ello, la TC tiene un papel crucial en el diagnóstico correcto del SOI. La TC identifica y confirma la presencia del SOI, además de identificar su localización y su causa.

En casos de sospecha clínica de SOI estudiados por TC, dada su capacidad de reconstrucción multiplanar y volumétrica, en el periodo postoperatorio inmediato puede identificar el material quirúrgico olvidado $y / o$ su efecto de masa ${ }^{2}$.

En los casos en que se identifica la compresa quirúrgica retenida o textiloma, en la intervención quirúrgica, al realizar la enterotomía y al identificarse la compresa quirúrgica retenida intraluminal como una masa, no hay evidencia de comunicación externa, pero sí se logra definir un trayecto fistuloso entre las asas intestinales proximales y distales, más allá de la masa. Aunque la migración intraluminal de la compresa quirúrgica retenida se ha descrito en informes médicos, la migración completa intraluminal sin datos característicos del SOI o de comunicación externa rara vez se han descrito ${ }^{5,6}$.

Se han descrito casos clinicos donde se ha encontrado la compresa quirúrgica retenida despues de una amplia diversidad de procedimientos quirúrgicos, pero la migración transmural es muy rara, infrecuente. El caso que nos ocupa representa una compresa quirúrgica retenida, que del espacio intraabdominal erosionó hacia la luz intestinal, con su subsecuente migración intraluminal.

En la publicación de Zantvoord ${ }^{7}$ se realizó una búsqueda sistemática en PubMed y se 
encontraron 64 casos de compresa quirúrgica retenida con migración transmural, principalmente después de una cirugía intraabdominal, con el sitio más frecuente de impactación del cuerpo extraño en el intestino (75\% de los casos), con dos casos en que la migración ocurrió hacia el estómago y siete hacia la vejiga. En esta misma serie, cinco casos que describen migración transdiafragmática, y tan solo cuatro casos en los que se describe la expulsión espontánea de la compresa quirúrgica retenida a través del recto. Hasta en un $93 \%$ de los casos de esta serie fue necesaria la reintervención quirúrgica para la resolución del cuadro7.

Una compresa quirúrgica retenida en la cavidad peritoneal es una complicación ocasional en la cirugía moderna. La compresa quirúrgica retenida puede migrar hacia la luz intestinal, pero el mecanismo es desconocido. Una gasa, de 4 por $4 \mathrm{~cm}$, se colocó en sitios diferentes dentro de la cavidad abdominal de ratas Wistar. A pesar de que no se identificó infección, la migración de la gasa hacia la luz intraluminal se demostró microscópicamente en 10 de las 36 ratas. En un análisis estadístico paralelo, con la estimación de Cutler-Ederer, se estableció que 0.58 de la migración ocurriría probablemente en 6 meses. La migración transmural de una compresa quirúrgica retenida, no solo se asoció con los diferentes sitios de la cavidad abdominal en los que se colocó ( $p=0.680)$, sino también con cuando se hizo o no una incisión seromuscular ( $p=0.306$ ). La hipótesis es, basada en el estudio micro y macroscópico, de 4 estadios: reacción a cuerpo extraño, infección secundaria, formación de masa y remodelación ${ }^{8}$.

Cuando hay cuerpos extraños dentro de la cavidad abdominal, el organismo trata de librarse de ellos, lo que provoca fenómenos defensivos, pero en algunas ocasiones se hacen inocuos, lo que hace imposible su detección ${ }^{1}$. Esta observación puede ser la explicación a la identificación de esta compresa después de una cirugía abdominal realizada años atrás 9 .

Hay dos formas de presentación de un cuerpo extraño en la cavidad abdominal, la primera está determinada por una reacción característica, que produce un cuadro clínico debido a una infección abdominal, con síntomas desde las primeras horas hasta los 6 meses posteriores. En otras ocasiones, se produce una reacción mesenquimatosa local ante estos cuerpos extraños asépticos, que se denomina inflamación encapsulante. En estos casos, el cuerpo extraño se mantiene por muchos años sin producir síntomas. Sin embargo, el deterioro de los órganos que afecta se va haciendo evidente con el transcurrir del tiempo.

El material quirúrgico extraño más frecuentemente olvidado («retenido») es la gasa. La presentación clínica de una gasa quirúrgica retenida puede variar desde una hallazco incidental en una proyección radiológica hasta una intensa reacción inflamatoria con SOI y perforación intestinal. El caso que nos ocupa, con el cuadro clínico con SOI y dolor abdominal agudo, ocurrió 3 años después de la intervención quirúrgica, en la que inadvertidamente se dejó una compresa. Aparentemente, la respuesta inflamatoria secundaria al cuerpo extraño condicionó un absceso alrededor que finalmente perforó hacia el trayecto intestinal, con la subsecuente migración intraluminal. Desde este sitio de perforación, la compresa migró dentro de la luz intestinal hasta el yeyuno, donde finalmente fue quitada quirúrgicamente. El paciente se recuperó sin complicaciones. 
En todo acto quirúrgico es indispensable realzar la importancia de llevar a cabo una revisión de la cavidad que incluya toda su totalidad, que se extienda a todos los cuadrantes, antes de dar por terminado el procedimiento ${ }^{10}$.

Aunque rara en la práctica diaria, se debe considerar la retención de compresas intraperitoneales en pacientes que se presentan con síntomas abdominales complejos posteriores a una cirugía abdominal previa.

No es raro que el diagnóstico de textiloma sea incidental y los estudios radiológicos sean de mucha ayuda para dilucidar su origen. Su reconocimiento temprano minimiza los riesgos quirúrgicos y contribuye a evitar complicaciones graves. El mejor abordaje de esta situación es su prevención, por lo que se debe utilizar rutinariamente material quirúrgico con material radioopaco. La vigilancia perioperatoria del material e instrumentos, así como el verificar su número con un conteo meticuloso y la revisión orientada del sitio quirúrgico al final del procedimiento son medidas sencillas esenciales ${ }^{3}$.

\section{FINANCIAMIENTO}

Ninguno.

\section{AGRADECIMIENTOS}

Los autores no han tenido fuente alguna de financiamiento para este artículo

\section{CONFLICTO DE INTERESES}

Los autores manifiestan no tener ningún conflicto de interés del manuscrito que se presenta a la revista Anales de Radiología México.

\section{RESPONSABILIDADES ÉTICAS}

Protección de personas y animales. Los autores declaran que para esta investigación no se han realizado experimentos en seres humanos ni en animales.

Confidencialidad de los datos. Los autores declaran que han seguido los protocolos de su centro de trabajo sobre la publicación de datos de pacientes.

Derecho a la privacidad y consentimiento informado. Los autores han obtenido el consentimiento informado de los pacientes y/o sujetos referidos en el artículo. Este documento obra en poder del autor de correspondencia.

\section{BIBLIOGRAFÍA}

1. Manzella A, Borba Filho P, Albuquerque E, Farias F, Kaercher J. Imaging of Gossypibomas: Pictorial Review. AJR. 2009;193:S94-S101.

2. Motta Ramírez GA, Oliva González Burgos O, Castillo Lima JA, Villalobos García E. Material quirúrgico olvidado: Gossypiboma, textiloma, gasoma. Anales de Radiología México. 2007;4:285-96.

3. Motta Ramírez GA, Rodríguez Treviño C, Ramírez Arias JL, Arciniega Ceballos ME, Alfonso Méndez M. Material quirúrgico retenido y acciones para evitar el error. ¿Debe operarse? Acta Médica Grupo Ángeles. 2009;7(3):158-64.

4. Pujahari AK. Decision Making in Bowel Obstruction: A Review. J Clin Diagn Res. 2016;10(11):PE07-12.

5. Kansakar R, Thapa P, Adhikari S. Intraluminal migration of Gossypiboma without intestinal obstruction for fourteen years. JNMA J Nepal Med Assoc. 2008;47(171):136-8.

6. Pujahari AK. Decision Making in Bowel Obstruction: A Review. J Clin Diagn Res. 2016;10(11):PE07-12.

7. Zantvoord $Y$, van der Weiden RM, van Hooff MH. Transmural migration of retained surgical sponges: a systematic review. Obstet Gynecol Surv. 2008;63(7):465-71.

8. Wattanasirichaigoon S. Transmural migration of a retained surgical sponge into the intestinal lumen: an experimental study. J Med Assoc Thai. 1996;79(7):415-22.

9. Silva CS, Caetano MR, Silva EA, Falco L, Murta EF. Complete migration of retained surgical sponge into ileum without sign of open intestinal wall. Arch Gynecol Obstet. 2001;265(2):103-4.

10. Dhillon JS, Park A. Transmural migration of a retained laparotomy sponge. Am Surg. 2002;68(7):603-5. 